\title{
La reforma del sector salud en Colombia y sus efectos en los programas de control de tuberculosis e inmunización
}

\author{
Health sector reform in Colombia and \\ its effects on tuberculosis control and \\ immunization programs
}

Carlos Ayala Cerna 1,2

Axel Kroeger 1

\footnotetext{
1 Liverpool School of Tropical Medicine. Pembroke Place, Liverpool L3 5QA, UK. A.Kroeger@liverpool.ac.uk

2 Instituto Colombiano de Medicina Tropical "Antonio Roldán Betancur". Carrera $43 A 52$ Sur, Instituto de Ciencias de la Salud, Sabaneta-Medellín, Colombia.
}

\begin{abstract}
This paper analyzes the effects of health reform in Colombia on public health programs at the local level, particularly the Expanded Program of Immunization (EPI) and the tuberculosis control program. The research was developed in three health districts in two States by analysis of documents, direct observation, and longitudinal follow-up of the transition process. The health districts were unprepared for the change, resulting in insufficient technical skills among staff as well as lack of awareness of important elements in the new system, associated with a reduction in immunization coverage and tuberculosis case detection. Structural problems in the new system included loss of immunization opportunities and lack of examination of tuberculosis contacts. The potentialities of the new system were the local development of a new organizational structure and the improvement of the information system; a limiting factor was exposure of the health system to local political interests. The general conclusion is that the transition period takes far longer than anticipated and requires a much greater information flow to both the local level and the community. There are transitory but also structural maladjustments that require a political response.
\end{abstract}

Key words Health Sector Reform; Tuberculosis; Immunization Programs

Resumen Este trabajo analiza las consecuencias de la reforma de salud en Colombia para los programas de salud pública a nivel local, particularmente el programa ampliado de inmunizaciones (PAI) y de control de tuberculosis. La investigación se desarrolló en tres municipios de dos departamentos, utilizando como técnicas de recolección de información: análisis de documentos, entrevistas semi-abiertas con funcionarios del sistema, observaciones directas y el acompanamiento del proceso de cambio. Los resultados fueron: no hay preparación en los municipios para el cambio, llevando al desconocimento de elementos importantes en el nuevo sistema, lo que se asocia con la reducción de coberturas del PAI y con la disminución de la detección de casos de tuberculosis. Problemas estructurales del nuevo sistema fueron: oportunidades perdidas de inmunización y de análisis de contacto de pacientes con tuberculosis. Las potenciales del nuevo sistema fueron el desarrollo de una nueva estructura organizacional municipal y el mejoramiento del sistema de información y la debilidad de ser expuesto a intereses políticos del nivel local. Se concluye que el período de transición del sistema es mucho más largo de lo previsto y requiere un mayor flujo de información hacia el nivel municipal y comunitario. Hay desajustes pasajeros y desajustes estructurales los cuales requieren respuestas políticas.

Palabras clave Reforma del Sector Salud; Tuberculosis; Programas de Inmunización 


\section{Introducción}

Colombia, un país con 32 departamentos y 1.050 municipios, con una gran heterogenidad cultural y étnica, inició los procesos de descentralización del Estado y del Sistema de Salud a mediados de la década de los 80 . En 1990, la Ley 10 y, en 1993, la Ley 60 dieron más impulso a la descentralización al suministrar la base legal para asignarle a los municipios la competencia operativa en la atención de salud del primer nivel de complejidad y, al gobierno departamental, los hospitales de segundo y tercer nivel de atención. La nueva constitución de 1991, otorgó mayores poderes a los municipios y fortaleció el papel de los departamentos; además, consideró la seguridad social como un derecho irrenunciable de todos los habitantes y un servicio público obligatorio que se prestará bajo la coordinación y el control del Estado (Ministerio de Salud, 2000a; PAHO, 1999). A partir de 1993, con la promulgación de la Ley 100 (Sistema General de Seguridad Social en Salud - SGSSS) (Franco, 1995), Colombia modificó el sistema de provisión de servicios de salud, entre ellos la seguridad social, cambiando el subsidio a la oferta por el subsidio a la demanda a través del aseguramiento con cobertura universal. La Ley 100 inició su implementación en enero de 1995.

El sistema prevee dos tipos de régimenes de afilición: el contributivo y el subsidiado. Al régimen subsidiado se afilia la población más pobre y vulnerable sin capacidad de pago. Las personas que no logran ser afiliadas a ninguno de los dos regímenes por ausencia de los oferentes o falta de recursos se les denominan participantes vinculados, siendo responsabilidad de las alcaldías de cada municipio la prestación de servicios de salud a esta población.

En el régimen contributivo se crearon las Empresas Promotoras de Salud (EPS) - los núcleos organizativos básicos del sistema - y para el segundo caso las Administradoras del Régimen Subsidiado (ARS). Una forma de ARS son las Empresas Solidarias de Salud que se forman como asociaciones o cooperativas. Las EPS y ARS deben planear, organizar y ejecutar las actividades necesarias para mantener el control de enfermedades y mantener la salud de los afiliados (la población asegurada). Ambas entidades y el alcalde en conjunto con la Secretaría de Salud Municipal contratan a las IPS (Intituciones Prestadoras de Servicios de Salud) y a las ESE (Empresas Sociales del Estado, básicamente los antiguos hospitales públicos) para la prestación de servicios a los asegurados. El nuevo sistema creó una separación de funciones: dirección (Consejo Nacional de Seguridad Social, Ministerio de Salud, Direcciones departamentales y municipales), financiamiento (FOSYGA - Fondo de solidaridad y garantía), administración (EPS, ARS), prestación de servicios (IPS, ESE) y vigilancia y control (Superintendencia de Salud y delegadas). El sistema prevee el Plan General de Beneficios que incluye, entre otros, el Plan de Atención Básica (PAB) y el Plan Obligatorio de Salud (POS). Acciones de salud pública se reflejan en el Plan de Atención Básica (PAB) que incluye acciones de promoción, prevención, vigilancia, desarrollo de la red de laboratorios, salud ambiental y control de vectores. Enfermedades de interés en salud pública incluyen enfermedades transmisibles de mayor importancia (tuberculosis, enfermedades transmitidas por vectores, enfermedades inmunoprevenibles y otras). Los municipios se encargan de las actividades de prevención y promoción a nivel colectivo y para tal fin desarrollan el PAB; además, diseñan un Plan Local de Salud con el fin de operativizar acciones en conjunto con otras entidades de la zona (EPS, ARS y ESE) (Ministerio de Salud, 2000; PAHO, 1999). Las EPS están obligadas a ofrecer los servicios de diagnóstico y tratamiento para los problemas de salud pública y también acciones de promoción y prevención a nivel individual, las cuales están definidas en el POS y las ARS en el Plan Obligatorio de Salud Subsidiado (POS-S).

Las nuevas competencias municipales en el sector salud con respecto a la salud pública se resumen en (Ministerio de Salud, 2000a; PAHO, 1999): dirigir el Sistema Local de Salud; garantizar (por contrato) la prestación de servicios para problemas de salud a los vinculados en el primer nivel de atención; realizar acciones de fomento de la salud y de prevención de la enfermedad y poner en marcha los programas para los beneficiarios del régimen subsidiado. Así, el Alcalde es responsable por poner en marcha las políticas del sector cuando el municipio ha cumplido con la "certificación" de requisitos establecidos por la ley. En el caso de que el municipio no esté certificado, la administración de estos recursos es responsabilidad del departamento.

¿Qué consecuencias tiene la reestructuración del Sistema de Seguridad Social anteriormente descrito para los programas de salud pública a nivel municipal? Ésta fue la pregunta principal de la siguiente investigación, que toma el control de tuberculosis y el programa ampliado de inmunizaciones (PAI) como ejemplo, teniendo los dos programas una importancia especial. 


\section{Metodología}

Se usó el método del estudio de caso (Yin, 1994) para analizar la situación en tres municipios, dos en un departamento con desarrollo económico avanzado (Antioquia) de la Costa Atlántica y uno en un departamento de poco desarrollo económico (Vichada) de la región oriental del país.

El estudio de base fué realizado entre septiembre de 1999 y marzo del 2000 con el fin de describir la situación de los servicios prestados en los programas de tuberculosis y PAI. En los departamentos se entrevistó a los representantes de las Direcciones Departamentales de Salud ( $n=9$ personas) y, en los municipios, a los gerentes locales de salud ( $n=6$ personas) y a los responsables de los programas de tuberculosis y PAI ( $\mathrm{n}=13$ personas), para identificar problemas y dificultades que se habían presentado en el desarrollo e implementación de actividades utilizando una lista de chequeo (entrevistas semiabiertas). Además, se visitó en cada municipio a los gerentes y directores de cada institución prestadora de servicios de salud: EPS, ARS, ESE e IPS ( $n=21$ personas) y se recolectó información estadística. Se analizaron las coberturas de inmunizaciones y los factores asociados con problemas del PAI; tendencias y problemas en la detección de casos de tuberculosis, el análisis de contactos y la búsqueda activa entre los sintomáticos respiratorios. Falló el intento de encontrar cifras e indicadores sobre el proceso y el funcionamiento del sistema municipal de salud por falta de un sistema de información adecuado.

En la segunda fase del estudio, se realizó el acompañamiento del proceso de cambio en uno de los municipios, durante 10 meses (marzo a diciembre 2000). El objetivo fue documentar potencialidades y limitaciones en el proceso de reforma con respecto a la gerencia del sistema municipal de salud y con énfasis en los programas de control de tuberculosis y del PAI. Un investigador se desplazaba al municipio en estudio una vez al mês, por 5 días, realizando entrevistas a profundidad con los actores principales ( $\mathrm{n}=10$ personas), discusiones de grupo (como parte de las reuniones mensuales del grupo gestor), observación directa (en las oficinas centrales y en los hospitales y Centros de Salud) y análisis estadístico.

\section{Resultados}

Situación organizativa y operacional del nuevo sistema (resultados del estudio base)

\section{- Contexto de los 3 municipios estudiados}

En la Tabla 1, se comparan las características de los tres municipios estudiados. Se observa que el municipio A, en la costa Atlántica, es el de mayor desarrollo, con alto nivel de urbanización y porcentaje relativamente alto de población asegurada. En el otro extremo se encuentra el municipio $\mathrm{C}$, al oriente del país, con alto índice de población rural y bajo porcentaje de la población asegurada; no ha tenido aún la certificación, y la Dirección Departamental de Salud es la dependencia directa para la toma de decisiones. La capacidad resolutiva de la Secretaría de Salud en el municipio A es relativamente alta, la cual se refleja en el número de personal disponible ( $\sin$ tomar en cuenta sus destrezas gerenciales), y debería ser potencialmente alta en el municipio $C$ después de la certificación. La Secretaría en el municipio B prácticamente no opera, por falta de personal.

\section{- Dificultades comunes en la implementación del Sistema General de Seguridad Social en Salud}

\section{a) Capacidad organizativa}

La mayor dificultad reconocida por la mayoría de los entrevistados fue el no haber tenido una adecuada preparación acerca de las funciones y responsabilidades establecidas en la ley y cómo debían ser éstas asumidas por los municipios. Un comentario frecuente fue que existe un número excesivo de resoluciones y normas, que es difícil conocerlas todas. Este problema fue más grave para los funcionarios nuevos. Según las entrevistas entre directores de hospitales y en las Secretarías de Salud Municipal, el personal de los hospitales no tuvo la capacitación necesaria para asumir los cambios y enfrentar los retos del nuevo sistema al convertirse en ESE y entrar a competir con entidades privadas prestadoras de servicios de salud. Las instituciones privadas, en cambio, no contaban con la experiencia en actividades de promoción, prevención y atención de los eventos de salud pública. Los organismos de vigilancia y control tampoco han ejercido esta función adecuadamente, dejando a las EPS y ARS sin la adecuada supervisión y el seguimiento de las actividades y servicios que prestan. 
Tabla 1

Sinopsis de los tres municipios estudiados.

\begin{tabular}{|c|c|c|c|}
\hline & Municipio A (Costa Atlántica) & Municipio B (Costa Atlántica) & Municipio C (Oriente) \\
\hline Economía de la zona & $\begin{array}{l}\text { Importante producción bananera, } \\
\text { agricultura y ganadería }\end{array}$ & $\begin{array}{l}\text { Exportación de banano, } \\
\text { agricultura, ganadería comercio } \\
\text { y servicios }\end{array}$ & $\begin{array}{l}\text { Agricultura, pesca y producción } \\
\text { temporal de algodón }\end{array}$ \\
\hline Población total & 108.380 habitantes & 111.633 habitantes & 46.861 habitantes \\
\hline Nivel de urbanización & $83 \%$ urbano; $17 \%$ rural & $38,9 \%$ urbano; $61,1 \%$ rural & $10,2 \%$ urbano; $89,8 \%$ rural \\
\hline Migración y desplazamiento & $\begin{array}{l}\text { Duplicó población en los } \\
\text { últimos } 8 \text { años. Desplazamiento } \\
\text { por violencia }\end{array}$ & $\begin{array}{l}\text { Población desplazada } \\
\text { transitoriamente por violencia. } \\
\text { Luego se dirige al municipio A }\end{array}$ & Poco desplazamiento a casco urbano \\
\hline Certificación* & Sí & Sí & No \\
\hline Aseguramiento & $\begin{array}{l}35,3 \% \text { EPS; } 30,2 \% \text { ARS; } \\
34,4 \% \text { Municipio** }\end{array}$ & $\begin{array}{l}\text { 19,3\% EPS; } 35,5 \% \text { ARS; } \\
\text { 45,2\% Municipio** }\end{array}$ & $\begin{array}{l}15,9 \% \text { EPS; } 56,2 \% \text { ARS; } \\
27,9 \% \text { Municipio** }\end{array}$ \\
\hline $\begin{array}{l}\text { Capacidad resolutiva } \\
\text { de la Secretaría de Salud }\end{array}$ & Alta (con limitaciones) & Muy baja & Limitada \\
\hline $\begin{array}{l}\text { Personal de la Secretaría } \\
\text { de Salud }\end{array}$ & $\begin{array}{l}1 \text { Secretario de Salud; } 7 \text { personal } \\
\text { técnico; } 2 \text { secretarias }\end{array}$ & 1 Secretario de Salud; 1 secretaria & $\begin{array}{l}1 \text { Secretario de Salud; } 5 \text { personal } \\
\text { técnico; } 2 \text { secretarias }\end{array}$ \\
\hline
\end{tabular}

* Es un acuerdo entre el Municipio y el Departamento que permite ejercer la autonomía que ordena la constitución.

Para poder cumplir con la certificación se deben cumplir con requisitos preestablecidos por ley.

** Parar los no asegurados (vinculados).

EPS $=$ Empresas Promotoras de Salud; $A R S=$ Administradoras del Régimen Subsidiado.

\section{b) Monitoreo del sistema}

Al mismo tiempo, la legislación dejó aspectos de control fuera de las competencias del municipio, como es el manejo de la información de las actividades que realizan las EPS, ya que éstas no están obligadas a enviarla a la Secretaría de Salud Municipal, sino a la dirección departamental. Esta situación mencionada en las Secretarías Municipales de Salud debilita el papel del municipio como evaluador y controlador de las actividades que realizan los diferentes actores del sistema.

\section{c) Financiamiento}

El pago tardío de los aportes por parte de los empleadores - mencionado por los representantes de las EPS - ha entorpecido la prestación de los servicios de salud. Al suceder esto, la EPS no puede conseguir el monto del FOSYGA y, a su vez, éste no le reconoce la Unidad de Pago por Capacitación (UPC) para la prestación de servicios, generando exclusión del empleado. Algunos funcionarios de las ARS mencionaron que, los pagos del POS-S, en el proceso de llegar a las ARS y de éstas al IPS, pasan por una serie de re- trasos, por provenir de distintas fuentes, y están sujetos a muchos condicionantes, lo que genera demora en la prestación de servicios.

\section{d) Afiliación al seguro social}

En las zonas de violencia armada y en las zonas urbanas en las que se encuentra población desplazada, se presenta - según información obtenida en los municipios A y B - un problema para la afiliación de nuevos asegurados en cualquiera de los dos sistemas, contributivo o subsidiado, pero mucho más en el segundo. Esto se debe, en parte, a la carencia de la documentación exigida por el proceso administrativo para ser afiliado, ya que muchas personas desplazadas han perdido sus documentos al momento de la reubicación forzada (Existe un fondo especial del FOSYGA destinado a atención en salud de los deplazados. Un barrio con 25.000 desplazados en Cartagena, creó su propia ARS y recibió el subsidio).

\section{e) Política local}

Los alcaldes municipales, al asumir su cargo, no conocían sus responsabilidades de gestión 
y dirección del sistema de salud. Esto ha llevado a que algunos alcaldes hayan sido encarcelados, en otros municipios, por malos manejos de los fondos públicos luego de auditorías hechas por el Estado. La responsabilidad de manejar actividades de salud a partir de los municipios ha sido vista como una posibilidad de incrementar la popularidad de los grupos políticos en el poder. Sin embargo, el tema de la salud también ha sido usado por los grupos opositores para desacreditar la gestión de los alcaldes ante la población; ejemplo, durante la epidemia de dengue en el municipio A, las autoridades de salud hacían propaganda para la eliminación de deshechos así como para la protección del agua en la casa, pero los representantes de la oposición hacían un llamado para que la población hiciera reclamos sobre la pavimentación de las calles ya que, a su criterio, los charcos de agua formados en los hoyos de la carrete- ra sin pavimentar eran erróneamente percibidos como los criaderos del vector del dengue.

\section{- Implicaciones para el PAI}

En los tres municipios estudiados, se encontró disminución en las coberturas de vacunación despues de 1995 (Tabla 2; Figura 1). Este fenómeno fue tambien observado a nivel nacional. La Encuesta Nacional de Demografía y Salud del 2000 (Profamilia, 2000) compara las coberturas del PAI con las del año 1995. Bajaron las coberturas de todas las vacunas de $66 \%$ a $52 \%$ y de la triple viral de $84 \%$ a $71 \%$. Sólo las vacunas aplicadas al nacimiento se mejoraron, como la primera dosis de Antipolio (aumento de $33 \%$ al $66 \%$ ).

En 1999, esto fue particularmente grave, debido principalmente al bajo suministro de biológicos durante varios meses por dificulta-

Tabla 2

El programa ampliado de immunizaciones (PAI) y de control de tuberculosis durante la fase de transición

del sistema de salud en Colombia.

\begin{tabular}{|c|c|c|c|}
\hline Programa & Municipio A (Costa Atlántica) & Municipio B (Costa Atlántica) & Municipio C (Oriente) \\
\hline PAI & $\begin{array}{l}\text { Descenso de coberturas de inmunización } \\
\text { a partir de } 1995 \text { - excepto BCG (Figura 1) } \\
\text { Acceso restringido a servicios de } \\
\text { vacunación } \\
\text { - Aseguradoras (EPS, ARS) sólo cubren } \\
\text { su propia población } \\
\text { - IPS tienen pocas actividades } \\
\text { extramurales } \\
\text { - Conflicto armado obstaculiza } \\
\text { Falta de coordinación de las instituciones } \\
\text { por la Secretaria Municipal en años } \\
\text { anteriores } \\
\text { Subregistro de la población infantil (por } \\
\text { desplazados y migración laboral) resulta en: } \\
\text { - Falta de biológicos } \\
\text { - Coberturas exageradas (> 100\%) } \\
\text { Deficiente información sobre población } \\
\text { no vacunada }\end{array}$ & $\begin{array}{l}\text { Coberturas de inmunización bajas, } \\
\text { incompletas e irregulares (desde } 1996 \text { se } \\
\text { han vacunado sólo antipolio con } 80 \% \\
\text { cobertura en 1998) } \\
\text { Acceso restingido a servicios de } \\
\text { vacunación. Comparado con municipio A: } \\
\text { - Conflicto armado mas grave; hay más } \\
\text { desplazados } \\
\text { - Zonas rurales más extensas (Tabla 1) } \\
\text { - No hay medios de transporte } \\
\text { - Problemas de EPS/ARS e IPS igual } \\
\text { como en municipio A } \\
\text { No hay promoción y preparación de los } \\
\text { eventos extramurales de vacunación } \\
\text { Subregistro de la población infantil (ver } \\
\text { municipio A) } \\
\text { Falta de coordinación por Secretaría } \\
\text { Municipal (la cual es débil; Tabla 1) }\end{array}$ & $\begin{array}{l}\text { Coberturas de inmunización bajas (excepto } \\
\text { BCG); descenso de } 1994 \text { a } 1999 \text { antipolio } \\
\text { (de } 53 \% \text { a } 40 \% \text { ), DPT (de } 51 \% \text { a } 35 \% \text { ) } \\
\text { antisarampionosa (de } 51 \% \text { a } 0 \% \text { ) y } \\
\text { antitetánica para mujeres en la edad fertil } \\
\text { (de } 19 \% \text { a } 12 \% \text { ) } \\
\text { Acceso restingido a servicios de vacunación: } \\
\text { - Falta de autonomía administrativa de } \\
\text { hospitales y centros de salud obstaculiza } \\
\text { trabajo extra-mural } \\
\text { - Faltan vehículos, combustible } \\
\text { - Zonas rurales extensas (Tabla 1) } \\
\text { No hay promoción; falta de interés por } \\
\text { parte del personal; no hay supervisión } \\
\text { Deficiente actualización del censo } \\
\text { poblacional por promotores y equipos } \\
\text { multidisciplinarios. } \\
\text { Falta la facturación de actividades } \\
\text { Falta de coordinación por Secretaría } \\
\text { Municipal que depende del Departamento } \\
\text { (Tabla 1) }\end{array}$ \\
\hline $\begin{array}{l}\text { Control de } \\
\text { tuberculosis }\end{array}$ & $\begin{array}{l}\text { Disminución de número de enfermos } \\
\text { con TB en tratamiento (Figura 2) } \\
\text { Obstáculos para el exámen de los } \\
\text { sintomáticos respiratorios (EPS no } \\
\text { autorizan exámen de laboratorio y } \\
\text { restringen tiempo de consulta externa) } \\
\text { Búsqueda activa entre contactos es } \\
\text { obstaculizado } \\
\text { EPS/ARS financian el tratamiento del } \\
\text { caso encontrado, pero el PAB municipal } \\
\text { es responsable por el estudio de contactos }\end{array}$ & $\begin{array}{l}\text { Disminución de enfermos de TB } \\
\text { Situaciones como en municipio A; además: } \\
\text { - no hay medicamentos suficientes } \\
\text { Los trámites para conseguir insumos son } \\
\text { largos porque quedan centralizados en } \\
\text { la capital del departamento } \\
\text { Los exámenes de esputo de sospechosos } \\
\text { son realizados en el municipio vecino; } \\
\text { radiografías se toman en una clínica } \\
\text { privada del municipio B }\end{array}$ & $\begin{array}{l}\text { Disminución de enfermos de TB } \\
\text { Situación como en municipio B; además: } \\
\text { - no hay interés del personal en el programa } \\
\text { - no tienen muchas funciones } \\
\text { - no hay supervisión } \\
\text { Dificultades económicas de la secretaría } \\
\text { impiden el estudio de contactos }\end{array}$ \\
\hline
\end{tabular}

EPS $=$ Empresas Promotoras de Salud; ARS = Administradoras del Régimen Subsidiado;

IPS = Instituciones Prestadoras de Servicios de Salud; TB = Tuberculosis; PAB = Plan de Atención Básica. 
Figura 1

Coberturas de vacunación en menores de 1 año y mujeres en edad fértil (MEF) de 15 a 44 años.

Municipio A, 1990-1999*

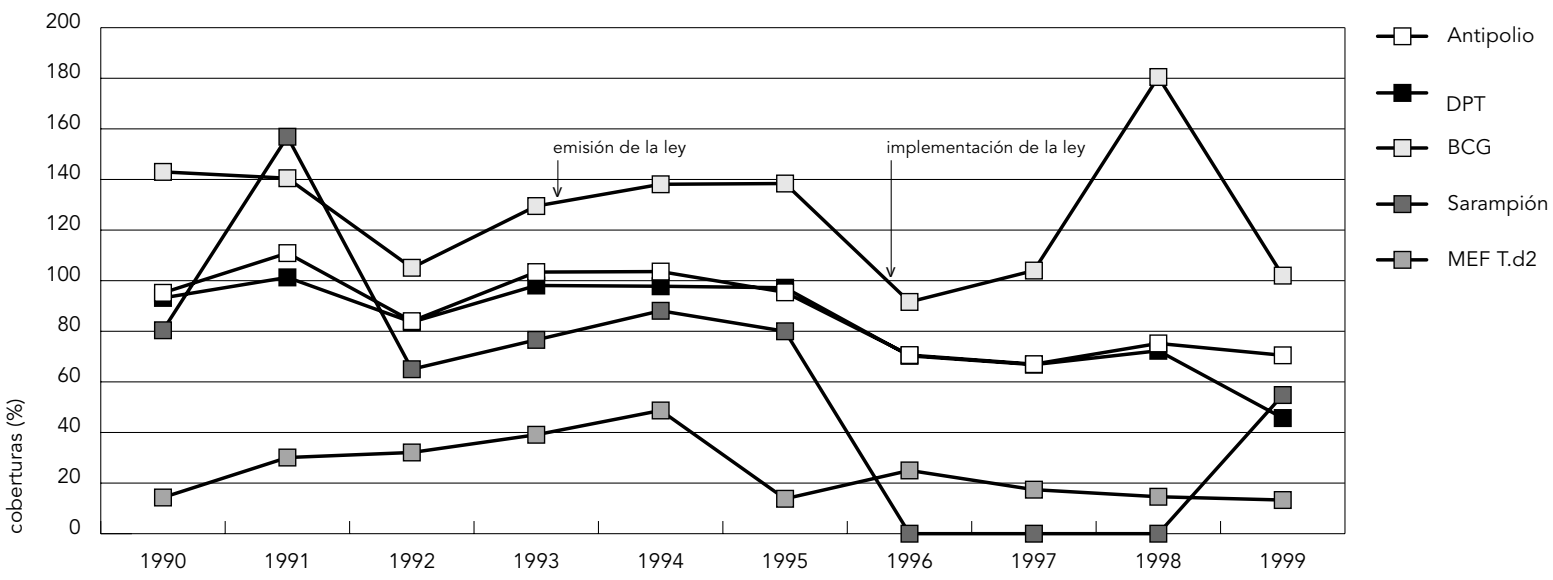

* Coberturas a diciembre 1999; T.d2 = dos dosis de tétanos.

Fuente: DDSA Oficina de Epidemiología.

des financieras en el Ministerio de Salud. Las coberturas de BCG en el municipio A fueron altas, con cierta variación, debidas al alto porcentaje de partos atendidos en las instituciones de salud. No se puede decir si éste es un efecto de la reforma, porque las atenciones de control prenatal y parto no fueron objeto de estudio en esta investigación.

También se han presentado dificultades de acceso a los servicios de vacunación, ocasionadas por los excesivos trámites y documentación exigida a los asegurados por parte de las EPS y ARS, particularmente en los municipios A y B. Un hecho común es que sólo se vacunan a los infantes asegurados por una determinada entidad, sin tomar en cuenta a los infantes asegurados por otra entidad o a los no asegurados, sí acaso éstos se encuentren en el mismo hogar. Además, hay pocas actividades extramurales realizadas por las IPS, hecho explicado por la falta de recursos y de autonomía para realizar actividades de vacunación extramuralmente. El nuevo sistema favorece la inmunización intra institucional, por ser más segura y por responder a la nueva forma de atención que pretende generar, a través del PAB, una mayor demanda de los servicios por la comunidad, en lugar de prestar en forma paternalista servicios seleccionados.

Adicionalmente, en los municipios rurales (B y C), la falta de transporte y la falta de interés por parte del personal (municipio $\mathrm{C}$ ) obsta- culizaron el trabajo extramural. El conflicto armado fue importante en los municipios A y B, generando miedos y recelo en la población, particularmente en las áreas rurales, la cual prefiere no acudir a los sitios de vacunación por temor a que aparezcan los actores del conflicto.

Se observa, en la Figura 1, que muchas coberturas en el municipio A fueron superiores al $100 \%$, debido al gran número de desplazados no registrados, lo que ha creado el subregistro de la población infantil y, además, un desabastecimiento de biológicos. Esto afecta justo a los desplazados, que tienen el mayor riesgo de desarrollar enfermedades infectocontagiosas, debido a las precarias condiciones en las que viven y al limitado acceso a los servicios de salud. Además, hay que señalar la poca coordinación y control de la Secretaría de Salud Municipal (que se estableció hace sólo 2 años) sobre las instituciones que prestan servicios de salud. El hecho que las EPS y ARS realicen actividades de vacunación sin coordinación hace que se pierda la información de la población no vacunada. En la Secretaría de Salud de los municipios B y C, la supervisión fue inadecuada a nivel local y departamental, por las dificultades económicas que impidieron un mayor desplazamiento a las zonas rurales. Había falta de autonomía administrativa de hospitales y centros de salud, particularmente en el municipio C, para las comisiones, combustible y mantenimiento de equipos y vehículos. 
- Implicaciones para el Programa de Tuberculosis

En los 3 municipios, se observó disminución en el número de pacientes en tratamiento (Figura 2). Algunas instituciones (IPS) realizan la búsqueda de sintomáticos respiratorios en las consultas, pero, cuando encuentran a un enfermo de tuberculosis, los tratamientos son solicitados a otra entidad encargada de la distribución de los medicamentos que está, a su vez, controlada por la Secretaría de Salud del municipio. Otras IPS remiten el paciente a otra entidad, donde se le inscribe al programa, y allí se le realiza el seguimiento y el control correspondiente. Los medicamentos son solicitados por la Secretaría de Salud a la capital del departamento. Los médicos de las IPS saben que toda persona con tos de más de 2 semanas debería ser remitida al laboratorio para el exámen de esputo. Sin embargo, tienen limitaciones por parte de las aseguradoras (EPS) para el uso de exámenes auxiliares por paciente. Hay límites por médico solicitante y por paciente atendido. El tiempo de consulta por paciente impuesto por las EPS también crea el problema de no se poder hacer una buena anamnésis o un exámen físico adecuado. Al mismo tiempo, las EPS requieren una serie de formularios por paciente atendido para hacer la facturación. Este proceso disminuye el tiempo destinado a la consulta. El estudio de contactos ha pasado a ser responsabilidad de la Secretaría de Salud como acciones a cargo del PAB, bajo la responsabilidad directa de la municipalidad y no de las EPS o ARS, pues, cuando éstas detectan un caso, se lo deben tratar a través de sus IPS contratadas. Algunas instituciones aseguradoras indagan si los contactos se encuentran afiliados a una EPS o ARS o si, no, son asegurados (vinculados), e informan a la institución correspondiente sobre la situación, para que realice el estudio respectivo de los contactos, o a la Secretaría de Salud Municipal, para los no asegurados. Dado que el seguimiento al estudio de contactos no se realiza, no es posible establecer cuantos de los casos nuevos están relacionados con el caso índice. En el municipio C, se observó que el personal no tiene interés en el programa, y hay una multiplicidad de funciones del mismo. Las dificultades económicas de la secretaría impiden la búsqueda activa de casos entre los contactos, y la falta de autonomía administrativa en el municipio dificulta la ejecución oportuna de los planes.
Figura 2

Tasas de incidencia de tuberculosis en todas las formas de los Departamentos en los que se encuentran los municipios A, B y C, 1993-1998.

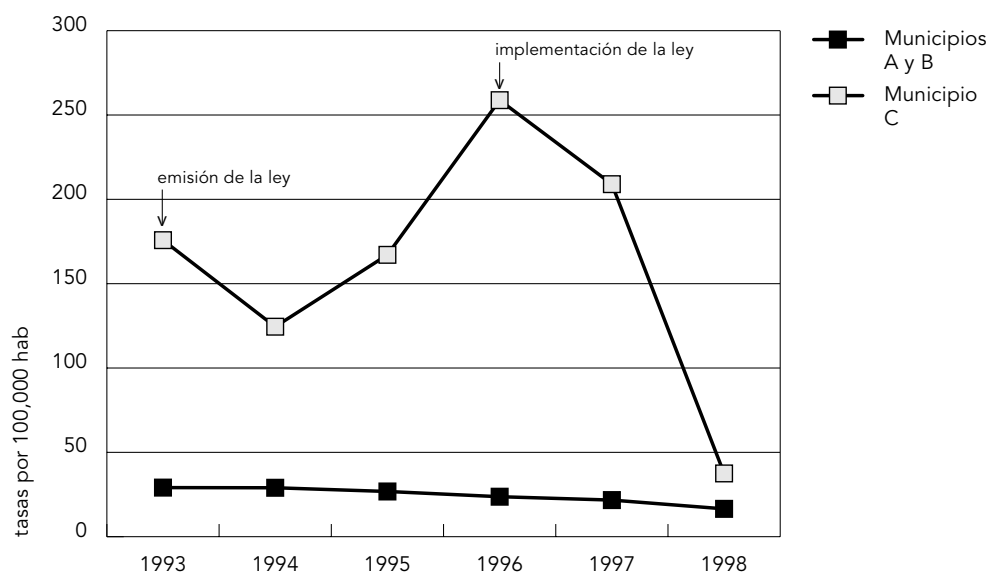

Fuente: Programa Patologías Infecciosas, Ministerio de Salud.

\section{Desafíos y desajustes en la}

operacionalización de la reforma a nivel

municipal (resultados de la fase de

acompañamiento en el municipio A)

- Desarrollo de la gerencia y coordinación de la Secretaría de Salud del municipio A

\section{a) Primer paso: análisis de contrataciones múltiples de IPS}

Durante esta fase, la Secretaría de Salud del municipio estaba adaptándose al nuevo sistema de gerencia. Se revisó la ley y los reglamentos vigentes y se identificaron los vacíos y las potenciales áreas de conflicto con los otros actores de salud en el municipio. Se elaboró una lista de las IPS y de otros prestadores privados de servicios de salud subcontratados por las EPS o declarados como independientes al sistema. Luego se analizaron los servicios ofrecidos; cotejando esta información con la información declarada por las EPS y ARS. Se identificaron 6 casos de doble o múltiple contratación de IPS lo que está restringido por ley por conllevar a una disminución de la calidad del servicio. Este análisis fue desarrollado con cuidado para mantener las buenas relaciones de trabajo existentes entre el personal de la Secretaría de Salud y las EPS, ARS e IPS. 


\section{b) Segundo paso: reestructuración de la organización}

Después de la revisión del actual organigrama de la institución, el personal de la Secretaría decidió como trabajo de equipo reestructurar la organización. Eso incluyó la determinación de los nuevos roles y responsabilidades de cada funcionario y el desarrollo del PAB. El nuevo sistema exige una organización más dinámica y con alta capacidad de decisión; pero ese proceso requiere formación y creación de una nueva mentalidad en el personal, lo que dificultó el cambio. Una barrera política para la reorganización fueron las imminentes elecciones municipales que retardaron la oportunidad para cambios importantes, dejando en algunos actores la sensación que no se trabaja en función del mejoramiento del sistema sino en función de la política local. Sin embargo, el personal de la secretaría estaba ganando prestigio con la formulación del Plan Local de Salud. Éste plantea actividades de salud pública, identifica la participación de los diferentes actores y toma en cuenta el financiamiento de los mismos.

\section{c) Tercer paso: la coordinación intra e intersectorial}

La epidemióloga de la Secretaría de Salud lideró el proceso de coordinación entre los diferentes actores en el municipio, lo que evidencia la importancia de las iniciativas individuales en la implementación de la Reforma. Ese proceso involucró a todas las entidades del municipio y creó un foro de discusión que se fué consolidando paulatinamente. Las actas tomadas en las reuniones permitían el monitoreo de los acuerdos, mostrando, en muchos casos, que el proceso de implementación de las decisiones fue lento debido a problemas de financiación. La misma epidemióloga implementó la edición de un boletín informativo con la opción de un suplemento sobre los roles y funciones de la Secretaría.

\section{- El fortalecimiento de los programas del PAl y de tuberculosis}

El PAI durante la fase de consolidación de la secretaría, logró un mayor grado de cobertura al apoyar el puesto de vacunación en el Hospital de segundo nivel como puesto de referencia de vacunación y de distribución de vacunas para las EPS y ARS. Antes de la reforma, el hospital era del primer nivel y muy conocido por la población como referente para las vacunaciones, lo que facilitó la concentración de la informa- ción en un sólo punto. El nivel departamental fortaleció el proceso y solicitó a todas las entidades usar un aplicativo informático único (PAISOFT) y canalizar la información a través del hospital a la Secretaría Municipal de Salud. Este aplicativo permitió una mejor facturación a las EPS y ARS ya que, aunque las vacunas son gratuitas, suministradas directamente por el Ministerio de Salud no lo son gastos con el mantenimiento de la cadena de frío e con insumos necesarios, como jeringas y algodón.

En el caso de tuberculosis, se trató de centralizar los procedimientos en el Hospital de primer nivel y de proceder como en el caso del PAI, pero eso no fué posible debido a la oposición de las APS y EPS. Dicho hospital atendía los casos de tuberculosis de la zona antes de la reforma y mantenía gran parte de su personal entrenado para el manejo de los casos y exámenes de laboratorio. Sin embargo, los laboratorios contratados por las EPS y ARS perdían un mercado y enfrentaron una reducción en la carga laboral de su personal, resultando en una posible disminución del mismo.

Dos factores adicionales frenaron el desarrollo de nuevas estrategias de control: (a) las epidemias de dengue y de malaria que se presentaron en el 2000, con más de mil enfermos en ambos casos, y que demandaron durante todo el tiempo del personal de la Secretaría y (b) la espera de la emisión de una norma (Resolución 412) por el Ministerio de Salud (2000b) que estandarizaba los procedimientos e intervenciones obigatorias para las EPS y ARS en la atención de enfermedades de interés en salud pública (Ministerio de Salud, 2000a), lo que permitiría a las secretarías de salud municipales exigir niveles de calidad a las EPS y ARS en la atención a los pacientes que presentan enfermedades infectocontagiosas. Sin embargo, la emisión de la resolución se había retrazado por más de dos años de lo estipulado por la ley y, hasta el final de la investigación, no se había emanado aún.

\section{- Mejora en el sistema de información}

Debido a la ausencia de formularios autorizados por el Ministerio de Salud para la recolección de información sobre tuberculosis y PAI, se usaron formularios temporales a la espera de la Resolución 412. En el caso del PAI, la Dirección Departamental propuso su propio aplicativo informático (PAISOFT, ver arriba). Para el caso de tuberculosis, se usaron formularios del Sistema de Vigilancia Epidemiológica en Salud (SIVIGILA), pero éstos sólo consideran el seguimiento del caso para su confirmación y 
posibles contactos. No hay formularios oficiales para el seguimiento terapéutico del paciente, y los formularios temporales son adaptaciones de formularios del sistema anterior.

\section{Discusión y conclusiones}

Colombia ha desarollado su reforma de salud y seguridad social según lineamientos establecidos (Cassels, 1995; PAHO, 1998) y sin apoyarse mucho en fondos foráneos (Barn \& Jessani, 2000). El país ha recibido comentarios favorables en la literatura (Bossert, 1998) y fue clasificado por la OMS como el país con el sistema de salud más equitativo del mundo en términos de garantizar a toda la población la accesibilidad financiera a los servicios de salud (WHO, 2000). Esta investigación ha mostrado que falta mucho en la implementación, aunque una extrapolación de los tres municipios estudiados al resto del país no es posible. Sin embargo, los tres casos analizados parecen ser bastante comunes. Representantes municipales del Norte y del Sur de Colombia que leyeron nuestros hallazgos encontraron situaciones similares en su propio contexto; reconfirmaron también la importancia de iniciativas individuales y en equipo para la implementación de la Reforma, similar a lo que observamos en el municipio A.
Algunas de las debilidades del nuevo sistema, identificadas en nuestras entrevistas, fueron mencionadas por otros autores, como la falta de supervisión de las EPS y ARS (Cardona, 1966; Céspedes et al., 2000; Pérez, 1997); el problema de los desplazados en zonas de conflicto de asegurarse oportunamente (Málaga et al., 2000; McGee et al., 1999) y el desconocimiento de los alcaldes en materias de salud pública (Kroeger et al., 2002). Algunos problemas similares fueron identificados en el programa de control de tuberculosis en Brasil, particularmente la falta de monitoreo de la implementación del sistema a nivel local y los pocos esfuerzos de capacitar y supervisar el personal operativo y, por ende, la reducción de detección de casos (Kritski \& Ruffino Netto, 2000; Ruffino Netto, 1999). En general, la literatura sobre las dificultades y potencialidades de la reforma en Colombia gira alrededor de aspectos económicos y de financiamiento (Herrera, 2000; Jaramillo, 1998; Morales et al., 1998; Paredes, 1999; Sanchez \& Nuñez, 1999). Este estudio ha identificado problemas en la implementación, algunos de ellos directamente relacionados a la reforma, otros debido a la situación política y social difícil del país (Tabla 3). No fue posible cuantificar los efectos de la reforma para los diferentes grupos de los asegurados y vinculados, pero fue posible evidenciar algunos problemas específicos para los más vulne-

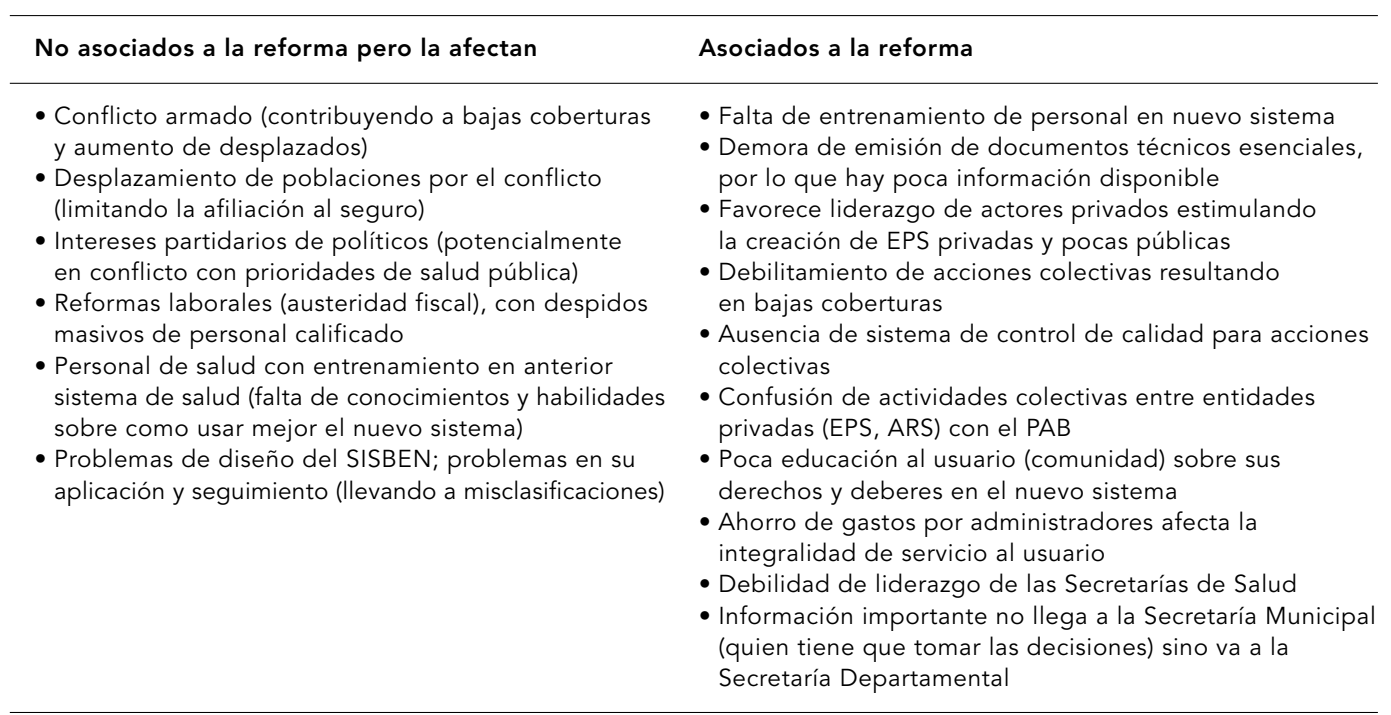

SISBEN = Sistema que determina la estratificación social de la población y con esto quien tiene acceso al régimen subsidiado; EPS = Empresas Promotoras de Salud; ARS = Administradoras del Régimen Subsidiado: $\mathrm{PAB}=$ Plan de Atención Básica, desarrollado por el municipio. 
rables, que son los afiliados al régimen subsidiado (por la complejidad del POS-S), y para muchos refugiados del conflicto armado ("desplazados"), debido a la dificultad de afiliarse al sistema de seguridad social.

Para aprender de las experiencias de la Reforma en Colombia, es útil distinguir entre desajustes temporales (que son asociados con el proceso de transformación) y desajustes definitivos e inherentes en la nueva estructura.

A los problemas pasajeros pertenecen el hecho que las Secretarías Municipales de Salud no tienen una estructura clara, con roles bien definidos, por lo que no pueden transmitir un mensaje preciso a las EPS, ARS e IPS. El ejemplo del municipio A mostró que el proceso de consolidación interna de la Secretaría no sólo mejora la imagen de la misma sino que sirve también como propaganda positiva para el alcalde ante la población de su municipio (Sanchez \& Nuñez, 1999). Un marco técnico referencial sobre las enfermedades sujetas a vigilancia, anunciado por el Ministerio de Salud, daría a la Secretaría de Salud Municipal un instrumento importante para los programas de salud pública (Málaga, 1999). El desconocimiento y la confusión de los actores locales por la enorme profusión de normas hacen imperativo el diseño y la implementación de un programa de capacitación (directo o a distancia). El personal que está implementando actividades bajo el nuevo sistema estuvo adaptado al anterior, evidente en el caso de la prestación extramural de inmunizaciones o en la inflexibilidad de usar fondos provenientes de diferentes fuentes (ejemplo del exámen de contactos de pacientes de tuberculosis con financiamiento del PAB o uso de fondos especiales para los desplazados). El personal requiere capacitación y tiempo, y la comunidad necesita in- formación y motivación para liberarse de los esquemas del sistema anterior.

A los problemas definitivos del nuevo sistema pertenece la complejidad del financiaminento y de los trámites requeridos (por ejemplo en el POS-S), que puede retrasar acciones urgentes. Otra área de preocupación es que los directores locales de EPS y ARS son, en su mayoría, gerentes de empresa y no tienen conocimientos de salud y mucho menos de salud pública. Con frecuencia persiguen como objetivo principal el ahorrar la mayor cantidad de dinero para la empresa, lo que puede perjudicar los programas de salud pública, como se ha mostrado para el control de tuberculosis y el PAI. Aceptan con dificultad que la inversión en un programa preventivo ahorrará recursos importantes. Este problema del sector privado ha sido mencionado a nivel global por Weill (2000), describiendo la tendencia de usar fondos asignados a salud pública para proyectos que son políticamente más atractivos.

El ejemplo de Colombia muestra las dificultades que implica el cambio radical del sistema de aseguramiento y las consecuencias de la descentralización para los programas de salud pública, los conflictos relacionados a las nuevas líneas de autoridad en el organigrama (quien tiene que responder a quien) y el nuevo rol de la comunidad que no está acostumbrada a liderar el proceso.

El proceso de la transición es largo (cerca de 7 años y aún no está terminado), requiere de ajustes políticos y un mejor conocimiento de su funcionamiento por parte de todos los actores del sistema. Queda aún por determinar, si una administración más centralizada podría favorecer la gerencia del sistema, como se describió para el Brasil (Kritsky \& Ruffino Netto, 2000).

\section{Agradecimientos}

\begin{abstract}
Agradecemos a todos los informantes de este estudio por su apoyo y colaboración para hacer un análisis crítico de la situación. El Ministerio de Salud en Bogotá apoyó en todo momento nuestro trabajo. Agradecemos también a Alejandro Castaño quien realizó gran parte del estudio basal con mucho profesionalismo y sensibilidad. Quisiéramos reconocer las contribuciones de Margarita Arboleda y del personal de la Secretaría de Salud que trabajó en el estudio de in-
\end{abstract}

tervención sin recibir ningún pago adicional a su salario. Sin su motivación no se hubieran podido terminar las investigaciones. Margarita Ronderos, Walter Flores y Sonia Díaz hicieron comentarios valiosos sobre los borradores de este trabajo. Agradecemos a la Comisión Europea, programa INCO-DEV por el apoyo financiero (Contrato IC18CT980339), como también al Consejo Británico en Colombia. 


\section{Referencias}

BARN, R. \& JESSANI, A., 2000. The role of the World Bank in international health: Renewed commitment and partnership. Social Science and Medicine, 50:183-184.

BOSSERT, T., 1998. Analysing the decentralization of health systems in developing countries: Decision space, innovation and performance. Social Science and Medicine, 47:1513-1527.

CARDONA, H., 1966. Las paradojas de la ley $100 \mathrm{de}$ 1993. Revista de Salud Pública, Universidad de Antioquia, 13:47-50.

CASSELS, A., 1995. Health Sector Reform: Key Issues in Less Developed Countries. Geneva: World Health Organization.

CESPEDES, E.; JARAMILLO, I.;, MARTINEZ, R.; OLAYA, S.; REYNALES, J.; URIBE, C.; CASTAÑO, R.; GARZON, E.; ALMEIDA, C.; TRAVASSOS, C.; FERNANDO, B. \& ANGARITA, J., 2000. Efectos de la reforma de la seguridad social en Salud en Colombia sobre la equidad en el acceso y la utilización de servicios de salud. Revista de Salud Pública, Universidad de Antioquia, 20:9-19.

FRANCO, A., 1995. La ley 100 de 1993, la salud pública y la seguridad social en Colombia. Revista de Salud Pública, Universidad de Antioquia, 13:20-41.

HERRERA, T., 2000. Régimen subsidiado de salud: Evolución y problemática. Revista de Salud Pública, Universidad de Antioquia, 20:21-27.

JARAMILLO, P., 1998. ¿Hay recursos para los más pobres? Análisis de la situación financiera del régimen subsidiado. Revista Vía Salud, Gestión, Calidad y Logros, 6:15-23.

KRITSKI, A. L. \& RUFFINO NETTO, A., 2000. Health sector reform in Brazil: Impact on tuberculosis control. International Journal of Tuberculosis and Lung Diseases, 4:622-626.

KROEGER, A.; ORDOÑEZ-GONZALEZ, J. \& AVIÑA, A., 2002. Malaria control reinvented: Health sector reform and strategy development in Colombia. Tropical Medicine and International Health, 7: 450-458.

MALAGA, H., 1999. Perspectivas de la epidemiología en la reforma de la seguridad social. Revista de Salud Pública, Universidad de Antioquia, 19:57-67.
MALAGA, H.; LA TORRE, M.; CARDENAS, J.; MONTIEL, H.; SAMPSON, C.; DE TABOADA, M.; GRANADOS, R. \& PASTOR, D., 2000. Equidad y reforma en salud en Colombia. Revista de Salud Pública, Universidad de Antioquia, 20:193-219.

McGEE, R., 1999. Technical, Objective, Equitable and Uniform? A Critique of the Colombian System for the Selection of Beneficiaries of Social Programmes, SISBEN. Brighton: University of Sussex.

MINISTERIO DE SALUD, 2000. Definición del Sistema de Seguridad Social en Salud. Santafé de Bogotá: Ministerio de Salud.

MINISTERIO DE SALUD, 2000. Resolución 00412. Santafé de Bogotá: Ministerio de Salud.

MORALES, L.; GIDEON, U.; PLAZA, B. \& BLOOM, E., 1998. La evasión en el sistema de salud de Colombia. Revista Vía Salud, Gestión, Calidad y Logros, 4:35-41.

PAHO (Pan-American Health Organization), 1998. Cooperation of the Pan American Health Organization in the Health Sector Reform Process. Washington, DC: PAHO.

PAREDES, N., 1999. ¿Qué está pasando con el régimen subsidiado? Revista Vía Salud, Gestión, Calidad y Logros, 9:18-22.

PEREZ, F., 1997. La autofinanciación de los hospitales públicos. Revista Vía Salud, Gestión, Calidad y Logros, 2:17-27.

PROFAMILIA (Asociación Probienestar de la Familia Colombiana), 2000. Salud Sexual y Reproductiva. Resultados Encuesta Nacional de Demografía y Salud. Santafé de Bogotá: PROFAMILIA.

RUFFINO NETTO, A., 1999. Impacto da reforma do setor saúde sobre os serviços de tuberculose no Brasil. Boletim de Pneumologia Sanitária, 7:7-18.

SANCHEZ, T. \& NUÑEZ, J., 1999. La ley 100/93 sí llegó a las personas de menores ingresos. Revista Vía Salud, Gestión, Calidad y Logros, 9:6-12.

WEILL, D. E. C., 2000. Advancing tuberculosis control with reforming health systems. International Journal of Tuberculosis and Lung Diseases, 4:597-605.

WHO (World Health Organization), 2000. World Health Report 2000. Geneva WHO.

YIN, R. K., 1994. Case Study Research. Design and Methods. 2nd Ed. Thousand Oaks: Sage Publications.

Recibido el 7 de febrero de 2002

Versión final presentada el 12 de junio de 2002

Aprobado el 27 de junio de 2002 